\title{
Long-term Stable Cationic Solid Lipid Nanoparticles for the Enhanced Intracellular Delivery of SMAD3 Antisense Oligonucleotides in Activated Murine Macrophages
}

\author{
Su-Eon $\mathrm{Jin}^{1}$ and Chong-Kook Kim ${ }^{1,2}$ \\ ${ }^{1}$ College of Pharmacy, Seoul National University, Seoul, Republic of Korea \\ ${ }^{2}$ College of Pharmacy, Inje University, Gimhae, Gyeongnam, Republic of Korea
}

Received, April 4, 2012; Revised, July 23, 2012; Accepted July 24, 2012; Published, July 27, 2012.

\begin{abstract}
Purpose: Long-term stable cationic solid lipid nanoparticles (cSLNs) were formulated to transfer SMAD3 antisense oligonucleotides (ASOs) into the cells to enhance the intracellular activity of the ASOs. The SMAD3 ASOs were designed to block the inflammatory processes linked to TGFB/SMAD3 pathway. Methods: The cSLN formulation was prepared by high-pressure homogenization method composed of 1,2-dioleoyl-3-trimethylammonium propane (DOTAP), dioleoylphosphoethanolamine (DOPE), Tween 20, and tricaprin as a solid lipid core $(1: 1: 1: 1.67, \mathrm{w} / \mathrm{w})$. The size and the zeta potential of the prepared cSLNs were measured by light scattering. The cSLN/ASO complexes were generated and introduced into the murine macrophage cells. After the treatment of the complexes, the cellular uptake of the complexes was determined by flow cytometry and the intracellular activity of SMAD3 ASOs from the complexes was evaluated by western blotting of SMAD3. In addition, TGF $\beta 1$, an upstream molecule of TGF $\beta / S M A D 3$ pathway, was monitored by ELISA. Results: The nano-scale sized cSLNs were positively charged and physically stable at $4^{\circ} \mathrm{C}$ during the storage up to 24 months. The uptake efficiency of the cSLN/ASO complexes into macrophage cells was enhanced up to $80 \%$ without cytotoxicity. After the treatment of the cSLN/ASO complexes, SMAD3 as well as TGF $\beta 1$ was significantly suppressed based on the SMAD3 ASO activity in the macrophage cells. In addition, the cSLN/ASO complexes prevented the morphological change to dendritic shape in the activated macrophage cells. Conclusion: These results suggest that the cSLNs have a potential to deliver the SMAD3 ASOs to intracellular compartments for the anti-inflammatory effect. The development of this strategy might lead to anti-inflammatory and anti-fibrotic therapies in immunological disorders.
\end{abstract}

This article is open to POST-PUBLICATION REVIEW. Registered readers (see "For Readers") may comment by clicking on ABSTRACT on the issue's contents page.

\section{INTRODUCTION}

SMAD3, a signaling molecule in the TGF $\beta / S M A D$ signaling pathway, is related to the inflammation that leads to fibrosis and cancer $(1$, 2). It can mediate the effects of TGF $\beta$ through the SMAD signaling pathway, which is consistently overexpressed with TGF $\beta$ in these disease states. Previous studies have shown that SMAD3 plays a crucial role in controlling cellular inflammatory responses (3). In particular, SMAD3 also has predominantly pro-inflammatory and pro-fibrotic activity (4). Thus, antisense oligonucleotides (ASOs) as SMAD3 inhibitors can alter the response to inflammation process, which is a primary cause of morbidity and mortality in human diseases such as cancer and fibrosis in the major organs $(2,5)$.

The ASOs have been investigated as a 'magic bullet' to treat several diseases since the 1970s (6, 7 ), which are designed to specifically hybridize to heterogeneous nuclear RNA or mature mRNA sequences for the inhibition of the expression of numerous genes. Although the ASOs are a specific inhibitor in the post-translational modulation, formivirsen (Vitravene ${ }^{\mathbb{B}}$ ), only one drug, has been approved by the FDA for the therapeutics use in ocular cytomegalovirus retinitis of AIDS patients (8). For the major obstacle of the achievement of the effective ASOs, it is difficult to transfer the ASOs to their eventual sites of action due to their large molecular weight, highly negative charge, rapid degradation by nuclease, etc.

Corresponding Author: Prof. Chong-Kook Kim, College of Pharmacy, Seoul National University, 1 Gwanak-ro Gwanakgu, Seoul, Republic of Korea; College of Pharmacy, Inje University, Gimhae, Gyeongnam, Republic of Korea Email: ckkim@snu.ac.kr, ckkim@inje.ac.kr 
The poor delivery efficiency of the ASOs into target cells can be overcome by using cationic lipids $(9,10)$, which are developing cationic lipidbased carriers with the structural modification (11, 12). Solid lipid nanoparticles (SLNs) have been recently developed as an alternative delivery system due to their improved physical stability based on the core of solid lipid inside the SLNs (13). Moreover, the cationic SLNs (cSLNs) have a significant potential to carry nucleic acid therapeutics in the use of cationic lipids (e.g., DOTAP) (Figure 1a) $(14,15)$. These nano-sized cSLNs can be produced and used to successfully generate the lipoplexes via charge-charge interaction (Figure 1b) to overcome the low binding capacity with the ASOs and the low uptake efficiency of the ASOs to target cells to have an activity.
We hypothesized that the cSLN-based ASOs against SMAD3 would alleviate the inflammation process via blocking the TGF $\beta / S M A D 3$ pathway. To have a therapeutic potential of the ASOs, those should be protected and specifically transferred to target cells. In this study, the long-term stable cSLNs were formulated as a delivery system of the ASOs to be pharmacologically active. The cSLN/ASO complexes were prepared by highpressure homogenization method and were characterized by the physicochemical properties such as size and zeta potential. In addition, the prevention effect of the cSLN/ASO complexes on the inflammatory process was examined in vitro based on blocking TGF $\beta /$ SMAD3 pathway. The suppression of SMAD3 as well as TGF $\beta 1$ was confirmed in the activated macrophage cells after the treatment of the cSLN/ASO complexes.

a

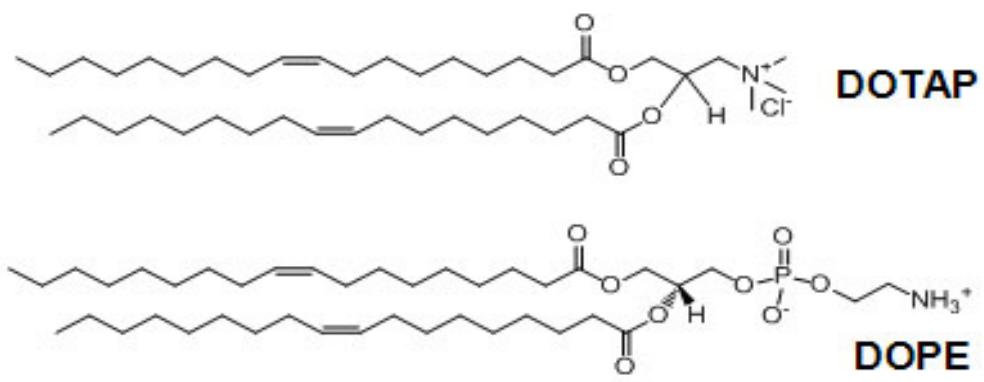

b

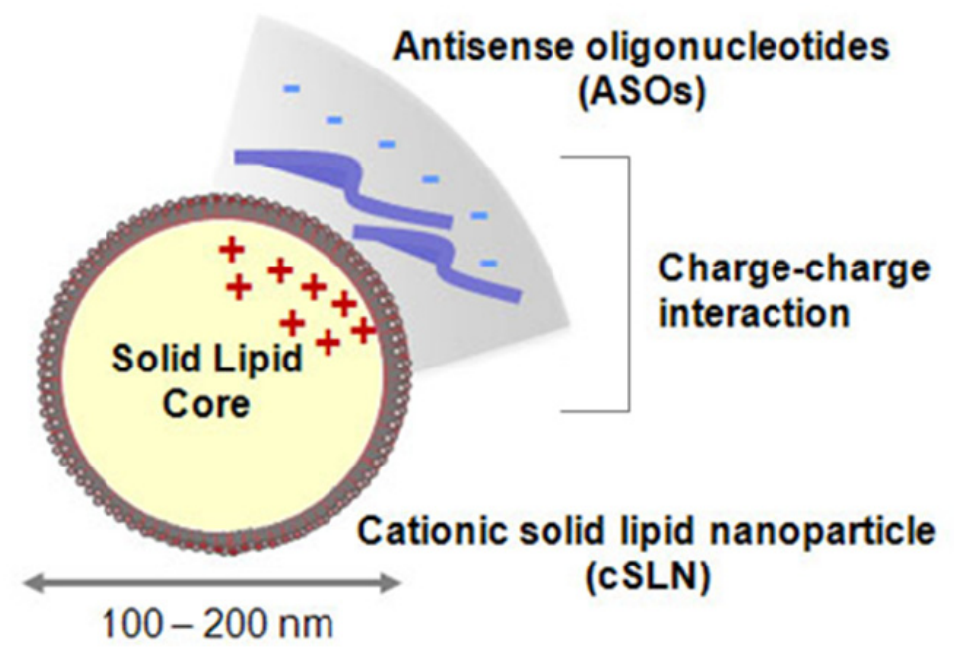

Figure 1. (a) Structures of the lipids used in this study, DOTAP (cationic lipid) and DOPE (helper lipid). DOTAP is a monoamine-based cationic lipid to make this formulation to have a positive charge. DOPE is a phosphatidylethanolamine-based helper lipid to stabilize the cationic lipid-including formulations. (b) Schematic diagram of the cSLNs and formation of the cSLN/ASO complexes via the charge-charge interaction. The cSLNs were nano-sized $(100-200 \mathrm{~nm})$ and positively charged to generate the complexes with the negatively charged ASOs. The immobilization of the ASOs on the cSLNs was based on the charge (positive from the cSLNs) - charge (negative from the ASOs) interaction. 


\section{MATERIALS AND METHODS}

\section{Reagents}

1,2-Dioleoyl-3-trimethylammonium propane (DOTAP) and dioleoylphosphoethanolamine (DOPE) were purchased from Avanti Polar Lipids. Inc. (Alabaster, AL, USA). Tween 20 and tricaprin (TC) were obtained from Sigma (St.Louis, Missouri, USA). Dulbecco's Modified Eagle Medium (DMEM), trypsin-ethylenediamine tetraacetic acid (EDTA), Lipofectin ${ }^{\circledR}$, and fetal bovine serum (FBS) were purchased from Invitrogen (Gibco-BRL, Invitrogen, San Diego, CA, USA). The ASOs designed to inhibit the translation of SMAD3 exhibited a high affinity for the translation region of the murine mRNA targets. All oligonucleotides (ONs) were phosphorothioate-modified at each end of three sequences to increase their resistance to nuclease (underlined). The following sequences were utilized as SMAD3 ASOs: 5'TGGGCCTACTGTCCCAATGTCA-3' (22 mer). SMAD3 sense oligonucleotides (SOs) were used as controls. All phosphorothioate $\mathrm{ONs}$ and fluorescein isothiocyanate (FITC)-labeled ONs were synthesized by Bioneer (Daejeon, Korea). The ONs were dissolved in sterile water and stored at $-20^{\circ} \mathrm{C}$. Deionized water was used after filtration through a $0.2-\mu \mathrm{m}$ filter. All other chemicals were of reagent grade and were used without further purification.

\section{Preparation of cSLNs and cSLN/ASO complexes}

The cSLNs were prepared by a high-pressure homogenization method with slight modification (16). Briefly, DOTAP, DOPE, Tween 20, and TC were mixed at the ratio of $1: 1: 1: 1.67(\mathrm{w} / \mathrm{w})$ and dissolved in $1 \mathrm{ml}$ of tertiary butylalcohol. After rapid freezing in liquid nitrogen, mixtures were lyophilized in an Ultra 35 EL freeze-dryer (Virtis, USA). The finely dispersed cakes were obtained after overnight lyophilization, and were then put in a water bath. Preheated water was added to the melts, followed by sonication for $90 \mathrm{~min}$ until crude emulsions were obtained. These crude emulsions were homogenized for 5 cycles at 100 $\mathrm{MPa}$ using a high-pressure homogenizer (Emulsiflex EF-B3, Avestin Inc., Canada) wired with heating tape (Thermolyne). The formulating temperature was $50-55^{\circ} \mathrm{C}$. The cSLNs were produced by subsequent cooling of the homogenized emulsions in liquid nitrogen. The cSLNs were then thawed and stored at $4^{\circ} \mathrm{C}$ in the dark to protect against lipid peroxidation. The final concentration of the cSLNs was $4.4 \%$ of total lipids $(\mathrm{w} / \mathrm{v})$. For the preparation of the complexes, the cSLNs were appropriately diluted with filtered phosphate-buffered saline (PBS). The cSLN/ASO complexes were prepared by mixing the ASOs with the cSLNs at mass ratios of 2 to 16 (cationic lipid to ASOs, w/w) in PBS. The mixtures were incubated at room temperature for $20 \mathrm{~min}$ before use.

\section{Differential scanning calorimetry (DSC)}

Thermal analysis was performed to evaluate the physical state of the lipid core in the cSLNs compared with bulk TC. The cSLNs were lyophilized for thermal analysis. The thermal characteristics were determined using a differential scanning calorimeter (DSC Q-1000, TA Instrument, UK). Aliquots weighing at 5 to 10 $\mathrm{mg}$ were loaded in an aluminum pan and crimped with an aluminum lid. DSC was used to analyze the samples from 20 to $80^{\circ} \mathrm{C}$ at a heating rate of $2^{\circ} \mathrm{C} / \mathrm{min}$ nitrogen flow $(20 \mathrm{ml} / \mathrm{min})$.

\section{Measurement of size and zeta potential}

The particle size of the cSLNs and the cSLN/ASO complexes was determined by light scattering using an electrophoretic light scattering spectrophotometer (ELS-8000, OTSUKA Electronics, Japan) at a fixed angle of $90^{\circ}$ at room temperature.

The zeta potentials of the cSLNs and the cSLN/ASO complexes were analyzed using an electrophoretic light scattering spectrophotometer (ELS-8000) equipped with a standard cell. Prior to the measurement, the cSLNs were diluted with the filtered water. The system was used in the auto-measurement mode.

\section{Physical stability of cSLNs}

The physical stability of the cSLNs was determined by monitoring both particle size and polydispersity index (P.I.) during the storage. The cSLNs were stored at $4^{\circ} \mathrm{C}$ for 24 months. For the preparation of samples, the cSLNs from three batches were appropriately diluted with filtered water to be directly introduced to the light scattering spectrophotometer (ELS-8000). Method of measuring size was described above in 'Measurement of size and zeta potential'.

Gel retardation assay of cSLN/ASO complexes The complexation between the cSLNs and the ASOs was determined by gel retardation assay. To detect the ASO bands in the gel, the amounts of the ASOs were fixed at $2 \mu \mathrm{g}$. Ten $\mu \mathrm{l}$ of the ASOs and $15 \mu \mathrm{l}$ of the cSLNs were vortex-mixed in 25 $\mu 1$ of PBS and incubated for $20 \mathrm{~min}$. The mass 
ratios of the cSLNs to the ASOs were used from 2 to 16 (cationic lipid to ASOs, w/w). The aliquots of samples $(20 \mu \mathrm{l})$ were resolved onto a $1.0 \%$ agarose gel with ethidium bromide and visualized with the UV transilluminator (Chemi-Imager 4400, Alpha Innotech Co., CA, USA).

\section{Nuclease protection assay of cSLN/ASO complexes}

The cSLNs were mixed with $0.5 \mu \mathrm{g}$ of the ASOs at a cSLN/ASO ratio of $4(\mathrm{w} / \mathrm{w})$, followed by incubation at room temperature for $20 \mathrm{~min}$. For digestion by DNase I, the cSLNs were prepared in water instead of PBS to avoid a possible interference of salt on DNase I activity. The cSLN/ASO complexes were exposed to DNase I $(0.25 \mathrm{U}$ and $0.50 \mathrm{U})$ for $30 \mathrm{~min}$ at $37^{\circ} \mathrm{C}$. The ASOs and Lipofectin ${ }^{\circledR}$-based ASO complexes were used as controls. The ASOs were retrieved by phenol/chloroform extraction followed by ethanol precipitation. The ASOs were then visualized on a $1.0 \%$ agarose gel containing ethidium bromide under UV transillumination (Chemi-Imager 4400).

\section{Cell culture}

NIH-3T3 cells (mouse embryonic fibroblasts) and RAW264.7 cells (mouse macrophage cells) were obtained from the American Type Culture Collection (ATCC, Rockville, MD, USA), which were grown in DMEM supplemented with $10 \%$ $(\mathrm{v} / \mathrm{v})$ heat-inactivated FBS, $100 \mathrm{U} / \mathrm{ml}$ penicillin, and $100 \mu \mathrm{g} / \mathrm{ml}$ streptomycin at $37^{\circ} \mathrm{C}$ in a humidified atmosphere of $5 \% \mathrm{CO}_{2}$.

\section{Flow cytometry}

Cells were seeded at $1.0 \times 10^{5}$ cells/well in 12 -well plates. After $24 \mathrm{~h}$ incubation, the cells were washed with PBS. The cSLN/SO complexes were generated with $2 \mu \mathrm{g}$ of FITC-labeled SOs at various weight ratios of the cSLNs to the SOs. The FITC-labeled, naked SOs were used as controls. The complexes were added to medium containing 5\% FBS, and treated cells were incubated in fresh medium at $37^{\circ} \mathrm{C}$ for $4 \mathrm{~h}$. After $24 \mathrm{~h}$, the cells were harvested and fixed with $1 \%$ formaldehyde in PBS. Cellular uptake of the complexes was determined using flow cytometry (FacsCalibur ${ }^{\mathbb{Q}}$, Becton Dickinson, USA) with CellQuestPro ${ }^{\circledR}$ software to count and calculate the percentage of fluorescent cells. FITC-positive events were determined using a standard gating technique. In brief, the gate was drawn along the maximal FITC intensity detected for the control samples. Total events (8,000 to 12,000 events) were selected from the list-mode data, which consisted of side scatter (SSC), forward scatter (FSC) and fluorescence emission. The percentage of the positive events was calculated as the events within the gate divided by total number of events, followed by the subtraction of the percentage of positive events in the control samples.

\section{MTT assay}

The cytotoxicity of the cSLN mass ratiodependent cSLN/SO complexes and the fixed cSLN ratio-based SOs, cSLNs, and cSLN/SO complexes was determined by MTT assay. First, the cell viability of the cSLN/SO complexes was investigated at the ratios of 2 to 16 in RAW264.7 cells. The SOs were used at the fixed amount of 1 $\mu \mathrm{g}$ to prepare the cSLN/SO complexes. The cells were seeded onto a 48-well plate at a cell density of $1.0 \times 10^{4}$ cells $/$ well. The cSLN/SO complexes were added to each well for $4 \mathrm{~h}$ and further incubated in fresh medium at $37^{\circ} \mathrm{C}$ for $24 \mathrm{~h}$. MTT (3-(4,5-dimethylthiazol-2-yl)-2,5-diphenyltetrazolium bromide) solution (5 $\mathrm{mg} / \mathrm{ml}$ in PBS) was added to each well, followed by incubation at $37^{\circ} \mathrm{C}$ for $4 \mathrm{~h}$. Dimethyl sulfoxide (DMSO) was added to each well to dissolve the formazan after the medium was removed. The absorbance was measured at $540 \mathrm{~nm}$ and \% survival was determined by comparison with the control group. Next, we confirmed the cytotoxicity of the SOs, the cSLNs, and the cSLN/SO complexes in RAW264.7 cells with and without stimulation of lipopolysaccharide (LPS, Sigma). In this case, the fixed ratio 4 was used for the cSLN/SO complex formation. The cells were seeded onto a 48-well plate at a cell density of $1.0 \times 10^{4}$ cells/well. The SOs, the cSLNs, and the cSLN/SO complexes were added to each well for $4 \mathrm{~h}$ incubation. The treated cells were stimulated with or without LPS $(1 \mu \mathrm{g} / \mathrm{ml})$ for $20 \mathrm{~h}$. MTT solution was added to each well. After the incubation with MTT solution at $37^{\circ} \mathrm{C}$ for $4 \mathrm{~h}$, the medium was removed and DMSO was added to each well to dissolve the generated formazan. The absorbance was measured at $540 \mathrm{~nm}$ and cell viability \% was calculated by comparison with the control group. Cell viability $(\%)=\left[\mathrm{OD}_{540}(\right.$ sample $) / \mathrm{OD}_{540}$ (control) $] \times 100$

\section{The cSLN-based SMAD3 ASO delivery in activated macrophage cells}

\section{Treatment of cSLN/ASO complexes}

RAW264.7 cells were incubated on $24 \times 24 \mathrm{~mm}$ coverslips at a density of $1.0 \times 10^{5}$ cells $/ \mathrm{cm}^{2}$. After $24 \mathrm{~h}$ incubation, the cells were pretreated with the cSLN/ASO complexes for $4 \mathrm{~h}$, followed by 
incubation with $1 \mu \mathrm{g} / \mathrm{ml}$ of LPS for $20 \mathrm{~h}$. The cSLN/ASO complexes were prepared in DMEM containing 5\% FBS with and without LPS after the treatment of the cSLN/ASO complexes. Untreated cells were used as controls, which were incubated in DMEM containing 5\% FBS without complexes or LPS.

\section{Western blotting for SMAD3}

For analysis of SMAD3 by western blot, nuclear extracts were prepared by washing samples twice with ice-cold PBS, and resuspending in $400 \mu \mathrm{l}$ of lysis buffer (10 mM HEPES (pH 7.9), $1.5 \mathrm{mM}$ $\mathrm{MgCl}_{2}, 10 \mathrm{mM} \mathrm{KCl}, 1 \mathrm{mM}$ DTT, and protease inhibitor cocktail (Sigma)) on ice. After homogenization of the cells and centrifugation at $10,000 \mathrm{~g}$ for $20 \mathrm{~min}$ at $4^{\circ} \mathrm{C}$, the supernatants were removed and the pellets were resuspended in an extraction buffer (20 mM HEPES (pH 7.9), 1.5 $\mathrm{mM} \mathrm{MgCl} 2,0.42 \mathrm{M} \mathrm{NaCl}, 0.2 \mathrm{mM}$ EDTA, $25 \%$ $(\mathrm{v} / \mathrm{v})$ glycerol, $1 \mathrm{mM}$ DTT, and protease inhibitor cocktail) by gentle shaking for $30 \mathrm{~min}$. After centrifugation at $20,000 \mathrm{~g}$ for $10 \mathrm{~min}$ at $4^{\circ} \mathrm{C}$, the supernatants that contained the nuclear extracts were collected and stored in aliquots at $-70^{\circ} \mathrm{C}$. The levels of SMAD3 were monitored by western blotting. The protein concentration of supernatants was determined using Bio-Rad detergent-compatible microprotein assay with bovine serum albumin (BSA) as a standard protein. Ten-microgram aliquots of protein were separated by sodium dodecylsulfatepolyacrylamide gel electrophoresis (SDS-PAGE) and transferred to nitrocellulose membranes. The nitrocellulose membrane was incubated for $1 \mathrm{~h}$ in a blocking buffer (5\% nonfat milk in PBS) followed by incubation with the SMAD3 antibody (1:200, Zymed, San Francisco, USA).

\section{ELISA for TGFß1}

A sandwich enzyme-linked immunosorbent assay (ELISA) was used for the analysis of TGF $\beta 1$ as a manufacturer's instruction (Becton Dickinson, CA, USA). After homogenization of cells and centrifugation at $10,000 \mathrm{~g}$ for $20 \mathrm{~min}$ at $4^{\circ} \mathrm{C}$, supernatants were collected. Latent TGF $\beta 1$ in samples was activated by $1 \mathrm{M} \mathrm{HCl}$ and neutralized by $1.2 \mathrm{M} \mathrm{NaOH}$ in $0.5 \mathrm{M}$ HEPES. Samples were centrifuged at $1,000 \mathrm{~g}$ for $5 \mathrm{~min}$ at $4^{\circ} \mathrm{C}$, and the supernatants were stored at $-70^{\circ} \mathrm{C}$ until analysis. A sample volume of $100 \mu \mathrm{l}$ was to assess its concentration of TGF $\beta 1$. Optical densities were measured on microplate reader at a wavelength of $450 \mathrm{~nm}$ (Tecan Group Ltd., Switzerland). Data were analyzed against the linear portion of the generated standard curve at the range of 15.6 to $1,000 \mathrm{pg} / \mathrm{ml}$.

\section{Morphological analysis of activated macrophages}

After the treatment of complexes with LPS, the cells were incubated with Biodipy ${ }^{\circledR}-\mathrm{C} 5$ (Invitrogen) for $10 \mathrm{~min}$ and then were rinsed with PBS. The rinsed cells were observed using confocal microscopy (Leica Microsystems, Wetzlar, Germany) supplemented with an argonkrypton laser and equipped with a $200 \times$ magnification. The excitation wavelength was $488 \mathrm{~nm}$. The acquisitions were recorded as normal representations.

\section{STATISTICAL ANALYSIS}

All results are expressed as means \pm SD. Statistical analysis of the data was performed using Student's t-test and ANOVA. A $p$ value of less than 0.05 was considered significant.

\section{RESULTS}

\section{Thermal analysis}

DSC is widely used to examine the melting point and re-crystallization behavior of the cSLNs. Figure 2 shows DSC curves of the TC (Figure 2a) and the cSLNs (Figure 2b). An endothermic peak of the TC from the solid lipid core of the cSLNs was observed at $31^{\circ} \mathrm{C}$, which was largely depressed in this formulation (Figure 2b) compared with that of the TC (Figure 2a). In addition, the melting peak of the cSLNs was lower than that of bulk lipid because the TC in the core of the cSLNs was presented as nano-sized crystals of solid lipid. It suggests that the cSLNs were well-formulated and successfully prepared in this study.

\section{Physical stability of cSLNs}

To evaluate the physical stability of the cSLNs, we monitored the particle size and the P.I. of the cSLNs during the storage (Figure 3a). The cSLNs themselves were nano-sized $(110-125 \mathrm{~nm})$ with small P.I. $(<0.2)$ to homogeneously distribute in solvent. The particular aggregates were not detectable during the storage. The particle size and the P.I. of the cSLNs were maintained at $4^{\circ} \mathrm{C}$ of dark place for 24 months.

Size and zeta potential of cSLNs and cSLN/ASO complexes

To characterize the physicochemical properties of the cSLNs and the cSLN/ASO complexes, the particle size and the zeta potential were measured 


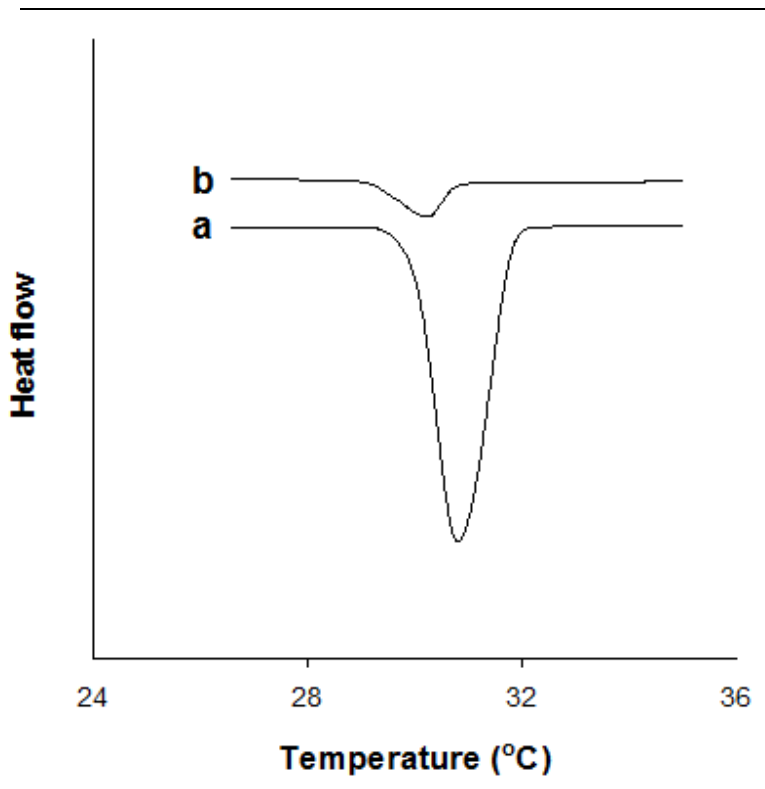

Figure 2 DSC curves of (a) the TC and (b) the cSLNs. The bulk TC, and the lyophilized cSLNs were analyzed from 20 to $80^{\circ} \mathrm{C}$ at a heating rate of $2^{\circ} \mathrm{C} / \mathrm{min}$ under a nitrogen flow of $20 \mathrm{ml} / \mathrm{min}$. The TC was used as a solid lipid core of this cSLN formulation, which was $31^{\circ} \mathrm{C}$ of the melting point. The peak from the cSLNs on the DSC results was remarkably depressed compared with that from the bulk TC because of the nano-crystalization of the cSLNs.

by light scattering. The cSLNs were nano-sized $(102 \mathrm{~nm})$ with a positive charge $(+42 \mathrm{mV})$ (Figure $3 \mathrm{~b}$ ). The mass ratios of 2 to 16 (cationic lipid to ASOs, w/w) were used to prepare the cSLN/ASO complexes. As the ratio of the cSLNs increased from 2 to 16 , the particle size decreased from 2.1 $\mu \mathrm{m}$ to $94 \mathrm{~nm}$ (Figure 3b). All complexes were smaller than $200 \mathrm{~nm}$ except the cSLN/ASO complexes at a ratio of 2 . The zeta potential increased from -22 up to $+36 \mathrm{mV}$ as the cSLN ratio increased from 2 to 16 . In addition, the zeta potential of the complexes reached a positive charge at a ratio of 4 suggesting the tightly immobilized ASOs on the surface of the cSLNs. In summary, Table 1 presents the particle size and the zeta potential of the ASO-unloaded and the ASO-loaded cSLNs at the fixed mass ratio of 4 (cationic lipid to ASOs, w/w).
Retardation of cSLN/ASO complexes

Gel retardation assay is used to confirm the complex formation between the cSLNs and the ASOs. The ASO band from the cSLN/ASO complexes was retarded on the gel as the mass ratio of the cSLNs increased from 2 to 16 , compared with that from the ASOs alone (Figure 4a). From the results, the bands from the complexes were retarded and disappeared in a mass ratio-dependent manner. In Figure $4 a$, the band from the complexes at the ratio of 4 was disappeared up to $97 \%$ based on the analysis of band density using Image $\mathrm{J}(\mathrm{NIH})$.

\section{Nuclease protection of cSLN/ASO complexes}

To examine the resistance of the complexes to enzymatic degradation, the cSLN/ASO complexes at the ratio of 4 were treated with DNase I, followed by gel electrophoresis to confirm the ASO band. In Figure 4b, the ASOs in the cSLN/ASO complexes were protected from DNase I at both concentrations of DNase I $(0.25$ $\mathrm{U}$ and $0.50 \mathrm{U}$ ). The ASOs were used as controls with and without the treatment of DNase I. After the treatment of the higher concentration of DNase I $(0.5 \mathrm{U})$, the band of the ASOs from the cSLN/ASO complexes remained while that from the ASOs was disappeared even at the lower concentration of DNase I. It suggests that the ASOs were protected from nuclease by the cSLNbased complex formation. In addition, the nuclease-protection effect of the cSLNs on the ASOs was superior to that of the commercial formulation, Lipofectin ${ }^{\circledR}$, which was utilized as a positive control.

\section{Enhanced cellular uptake of cSLN/SO complexes onto cells}

To monitor the cellular uptake of the cSLN-based complexes, the cSLN/SO complexes at the mass ratios varying from 2 to 16 were transfected into RAW264.7 cells and NIH-3T3 cells (Figure 5a). The cSLN/SO complexes were used instead of the cSLN/ASO complexes for the optimization of uptake potential of the complexes without antisense activity.

\begin{tabular}{|c|c|c|}
\hline & Mean diameter $(\mathrm{nm})$ & Zeta potential (mV) \\
\hline cSLNs & $101.9 \pm 4.3$ & $42.5 \pm 3.4$ \\
\hline $\begin{array}{l}\text { cSLN/ASO complexes } \\
\text { (ratio, 4) }\end{array}$ & $159.8 \pm 24.8$ & $5.3 \pm 2.1$ \\
\hline
\end{tabular}


a

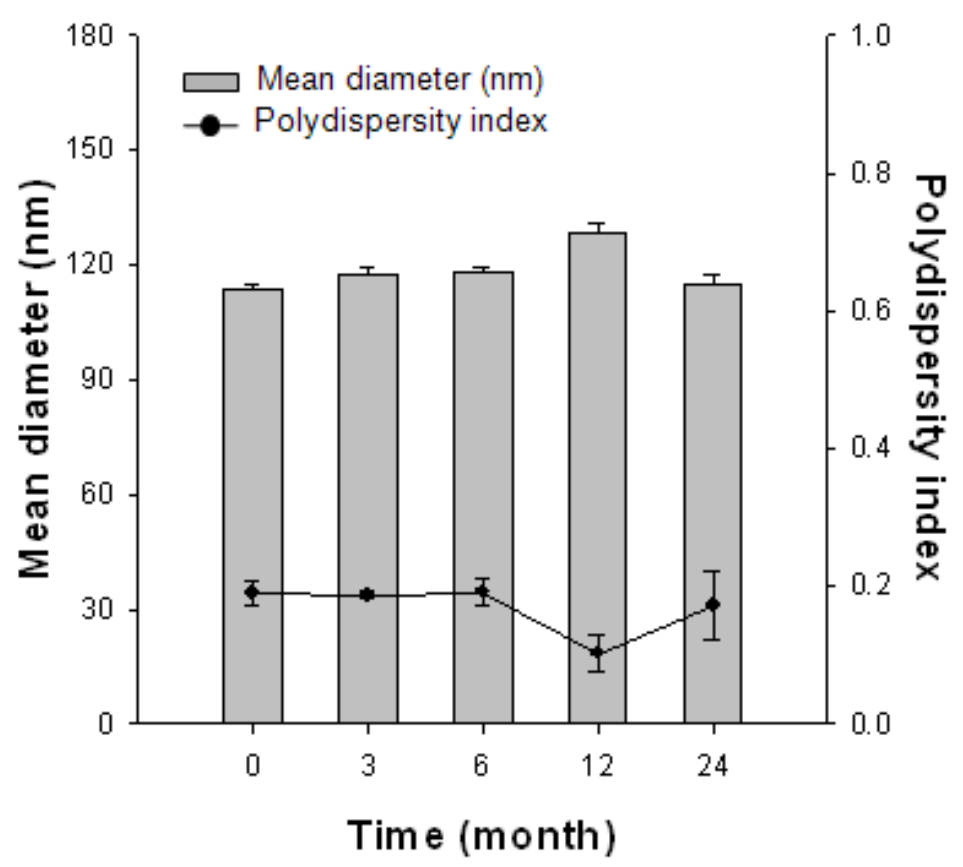

b

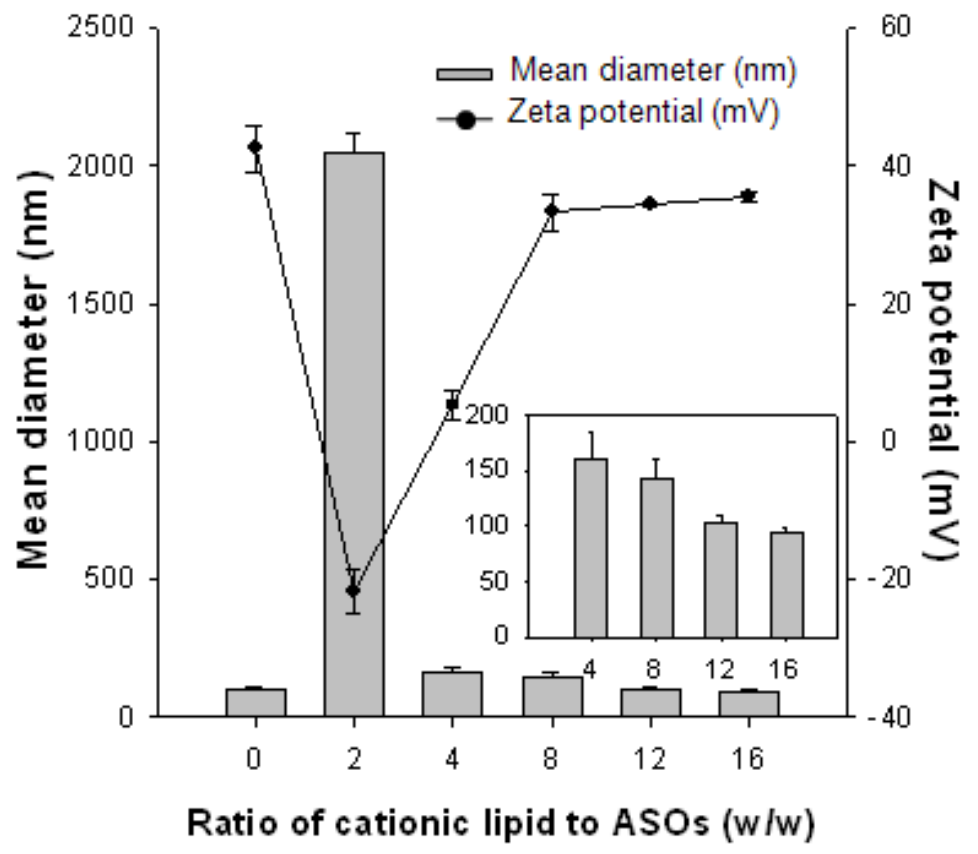

Figure 3 (a) Physical stability of the cSLNs. Size and polydispersity index (P.I.) of the cSLNs were monitored during the storage for 24 months at $4^{\circ} \mathrm{C}$. The results are expressed as the mean $\pm \mathrm{SD}(\mathrm{n}=3)$. (b) Size and zeta potential of the cSLN/ASO complexes. The cSLNs were prepared using cationic lipid, DOTAP $(1.2 \%$ of formulation, $w / v)$ at the mass ratios of 2 to 16 , and the ASO content was fixed at $2 \mu \mathrm{g}$. The cSLN/ASO complexes were generated prior to the measurements. The results are expressed as the mean $\pm \mathrm{SD}(\mathrm{n}=3)$. 


\section{a}

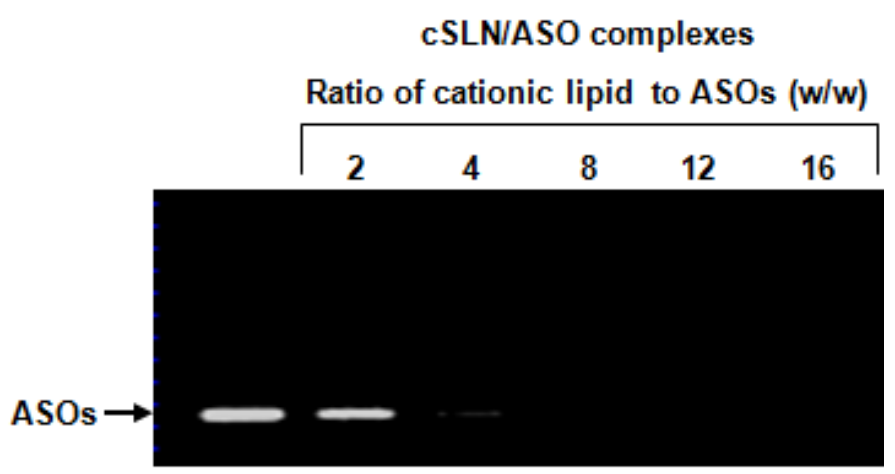

b

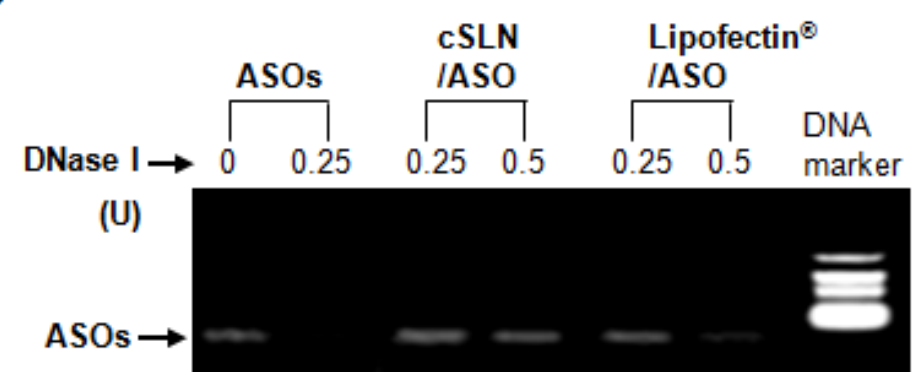

Figure 4. Generation of the cSLN/ASO complexes. (a) Retardation of the cSLN/ASO complexes. The ratios of the cSLN/ASO complexes were used from 2 to 16 (cationic lipid to ASOs, w/w). The ASOs were used at the fixed amount of $2 \mu \mathrm{g}$. The ASO bands from the cSLN/ASO complexes were retarded and disappeared in a cSLN mass-dependent manner. (b) Nuclease stability of the cSLN/ASO complexes. The ASO bands from the cSLN/ASO complexes were on the gel after the DNase I treatment. The complex ratio $4(\mathrm{w} / \mathrm{w})$ of the cSLNs was used with $0.5 \mu \mathrm{g}$ of the ASOs. Lipofectin ${ }^{\circledR}$ was used as a positive control.

Cells treated with the naked FITC-labeled SOs were used as controls. The uptake efficiencies at the ratios of $8-16$ were not significantly different, which were lower than that of a cSLN/SO ratio of 4 for both RAW264.7 cells and NIH-3T3 cells. The uptake efficiency of complexes at the ratio of 4 was maximized at both cells (RAW264.7 - 76\% and NIH3T3- 29\%). Figure $5 \mathrm{~b}$ presents the representative histogram of the $\mathrm{cSLN} / \mathrm{SO}$ complex (the ratio of 4, w/w)-treated RAW264.7 cells comparing with untreated cells showing over $80 \%$ of cellular uptake. This uptake efficiency of the cSLN/SO complexes was higher than that of Lipofectin ${ }^{\circledR}$-based SO complexes. In addition, the long-term stored cSLNs (24 months) could deliver the SOs to the cells. The uptake efficiency of the long-term stored cSLN-based complexes was comparable to that of the freshly prepared cSLN-based complexes. We selected the mass ratio of 4 of the cSLN/ASO complexes as the optimal ratio for further determination of the
SMAD3 ASOs based on this result of the enhanced uptake with several characterization studies mentioned above.

\section{Cytotoxicity of cSLN-based SO complexes}

The cytotoxicity of the cSLN/SO complexes were examined in RAW264.7 cells by MTT assay to confirm the optimized condition for the cellular delivery of the cSLN/ASO complexes. We monitored the cSLN ratio-dependent cytotoxicity of the cSLN/SO complexes and the toxicities of the cSLNs, the SOs, and the cSLN/SO complexes at the working concentration. Figure 6 a shows the cell viability of the cSLN/SO complexes $v s$ the mass ratios of the cSLN/SO complexes (cationic lipid to SOs, w/w). As the ratios of the complexes increased from 2 to 16 , the cell viability of the cSLN/SO complexes decreased from $98 \%$ to $58 \%$ (Figure 6a). The complexes at the ratios of 2 and 4 show the acceptable cell viabilities to use for the cellular delivery of the ASOs ( $>80 \%)$. 
a

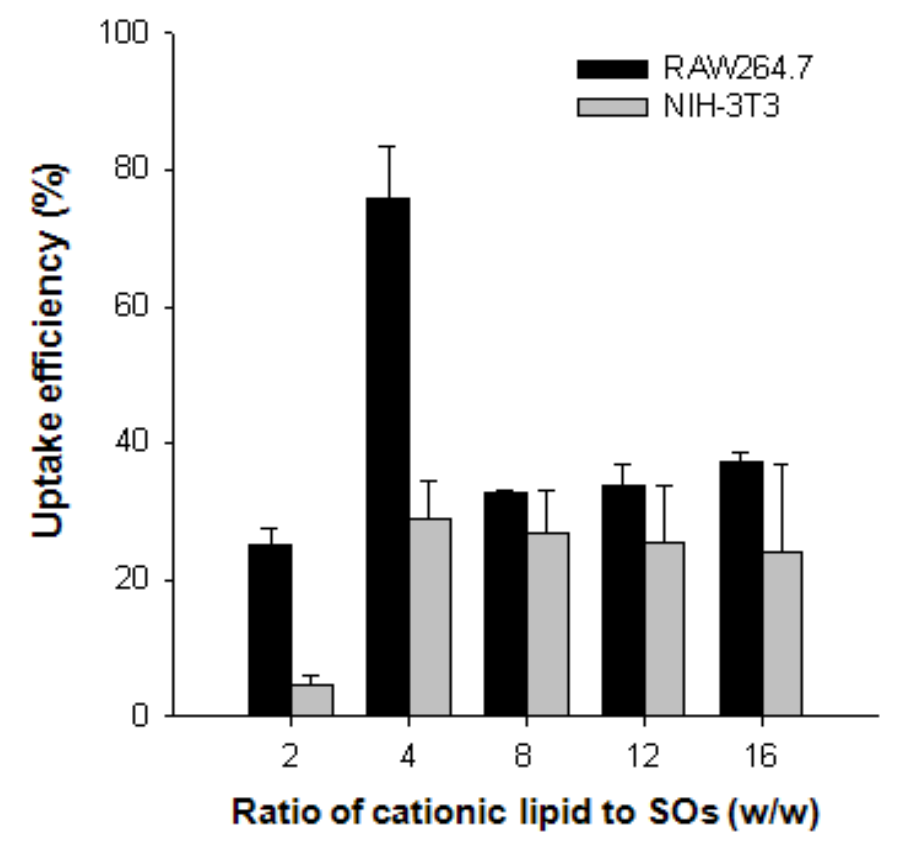

b

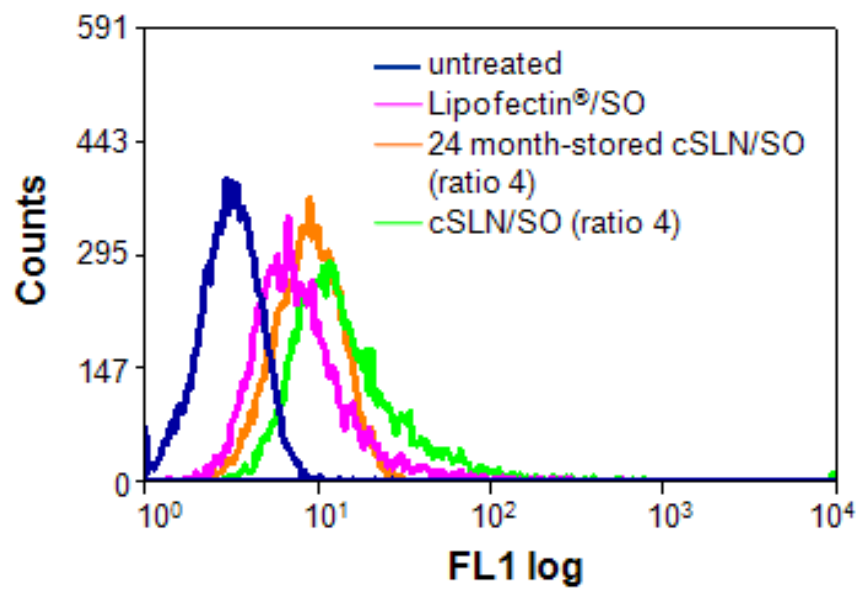

Figure 5. Uptake efficiency of the cSLN/SO complexes into the cells. (a) Uptake efficiency of the cSLN/SO complexes in RAW264.7 and NIH-3T3 cells. The FITC-labeled SOs were utilized at the mass ratios of 2 to 16 (cationic lipid to SOs, w/w). The results are expressed as the mean $\pm \mathrm{SD}(\mathrm{n}=3)$. (b) Representative flow cytometric histogram of the cSLN/SO complexes at the ratio of 4 in RAW264.7 cells. The cellular uptake of the 24 month-stored cSLN/SO complexes was also monitored. Lipofectin ${ }^{\circledR}$ was used as a positive control.

The toxicities of the cSLNs, the SOs, and the cSLN/SO complexes were monitored at the optimized working concentration in RAW264.7 cells. The relative cell viability (\%) was higher than $90 \%$ for these cells treated with and without LPS (Figure 6b). None of the complexes was cytotoxic at the concentration used in this study.
Intracellular activity of cSLN-based SMAD3 ASOs in the activated macrophages

Targeted inhibition of SMAD3 by cSLN/ASO complexes

After the LPS stimulation to induce the TGF $3 /$ SMAD signaling in RAW264.7 cells, the relative expression of SMAD3 in the nuclear 
extracts of cells was analyzed by western blotting. Figure $7 \mathrm{a}$ shows the relative band density of SMAD3/ $\beta$-actin from the cells after the treatment of the cSLN/ASO complexes. The SMAD3 significantly increased in the LPS-stimulated macrophage cells compared with that in the LPSuntreated macrophage cells by $17 \%$ (PBS - LPS $v s$ PBS + LPS, $p=0.00091$ ) (Figure 7a). The overexpression of SMAD3 in the activated macrophage cells was significantly inhibited by the cSLN/ASO complexes (PBS + LPS vs cSLN/ASO + LPS, $p=0.0086$ ). From the results, the cSLN-based SMAD3 ASOs can enhance the intracellular activity of the ASOs to inhibit the expression of target protein, SMAD3, with the optimized intracellular delivery of the ASOs in the activated macrophages.

\section{Effect of cSLN-based SMAD3 ASOs on the cellular level of TGFß1}

To examine the possible effects of the cSLN/SMAD3 ASO complexes on the upstream molecule in the signaling pathway, the level of TGF $\beta 1$ was monitored using ELISA. The cellular level of TGF $\beta 1$ was 4-fold increased by the LPS stimulation in RAW264.7 cells. After the treatment of the cSLN/ASO complexes, it was significantly down-regulated by the SMAD3 ASOs (Figure 7b).

\section{Morphological analysis}

To determine the effects of the SMAD3 ASOs on the inflammation, the morphological change of cells was examined in the LPS-stimulated RAW264.7 cells using microscopy (Figure 8). The RAW264.7 cells were stained by green fluorescence to clearly visualize whether the morphology of the cells was changed from circular shape to dendritic shape after the treatment of LPS (Figure 8a-d). After the LPS stimulation followed by the treatment of PBS (PBS + LPS), the dendritic shape of RAW264.7 cells was detected in Figure 8bb' and pointed by red arrows in Figure 8b'. After the treatment of the cSLN/ASO complexes (cSLN/ASO + LPS), the cells were maintained their circular shape (Figure 8dd') compared with the treatment of the cSLN/SO complexes (cSLN/SO + LPS) (Figure $8 \mathrm{cc}$ ). These results suggest that the inhibition of the morphological changes may correlate with the reduced expression of SMAD3 and TGF $\beta 1$. The cSLN-based SMAD3 ASOs can prevent the LPSinduced activation of macrophage cells, which occurred via the suppression of SMAD3 as well as TGF $\beta 1$.

\section{DISCUSSION}

The cSLNs have been developed as an alternative nanocarrier with the physical stability compared with the conventional formulations such as liposomes and emulsions. In addition, the cSLNs have been applied for the delivery of nucleic acid therapeutics from the previous reports $(13,16)$. Although the physical stability of the previous cSLNs was improved during the experiment, the transfection efficiency and/or the uptake efficiency of the cSLN-based nucleic acid therapeutics were generally lower than conventional formulations. Therefore, we report the physically stable cSLNs for the long-term storage to successfully transfer the SMAD3 ASOs into the cells. In this study, the size and the P.I. of the prepared stable cSLNs were maintained at $4^{\circ} \mathrm{C}$ up to 24 months. In addition, the cSLNs enhanced the uptake efficiency of the ASOs to the cells even at the low mass ratio of the cSLNs.

For the formulation of the cSLNs, the composition is one of key factors to generate a stable and nano-sized particular system (14). In the present study, the cSLNs were formulated to use as an intracellular delivery system of the ASOs based on the functional analysis of the components. The cSLNs were prepared by highpressure homogenization method, composed of DOTAP, DOPE, Tween 20, and TC. The structures of lipids of components were presented in Figure 1a. In this formulation, DOTAP was used to make this formulation positive, which is a monoamine-based cationic lipid (Figure 1a). The cationic lipids should be included into the cSLN formulation to generate the cSLN/ASO complexes based on the charge-charge interaction between the cSLNs and the ASOs (Figure 1b) (17, 18). In addition, DOTAP enhanced the transfection efficiency of nucleic acid-based therapeutics in the previous reports (19). DOPE is used for the enhanced stabilization of cationic lipid-based formulations and the ASO-loaded complex formation, which is called as a helper lipid (Figure 1a). It also has a function to enhance the cellular uptake of nanoparticles as it improves the association of the cSLNs to cell membrane (20). Tween 20 is a non-ionic surfactant which covers the particular interface of the cSLNs to form the nanoparticles in this study. TC (m.p. 31$32^{\circ} \mathrm{C}$ ), the solid lipid core, can inhibit the aggregation of nanoparticles to make this formulation stable (21). 
a

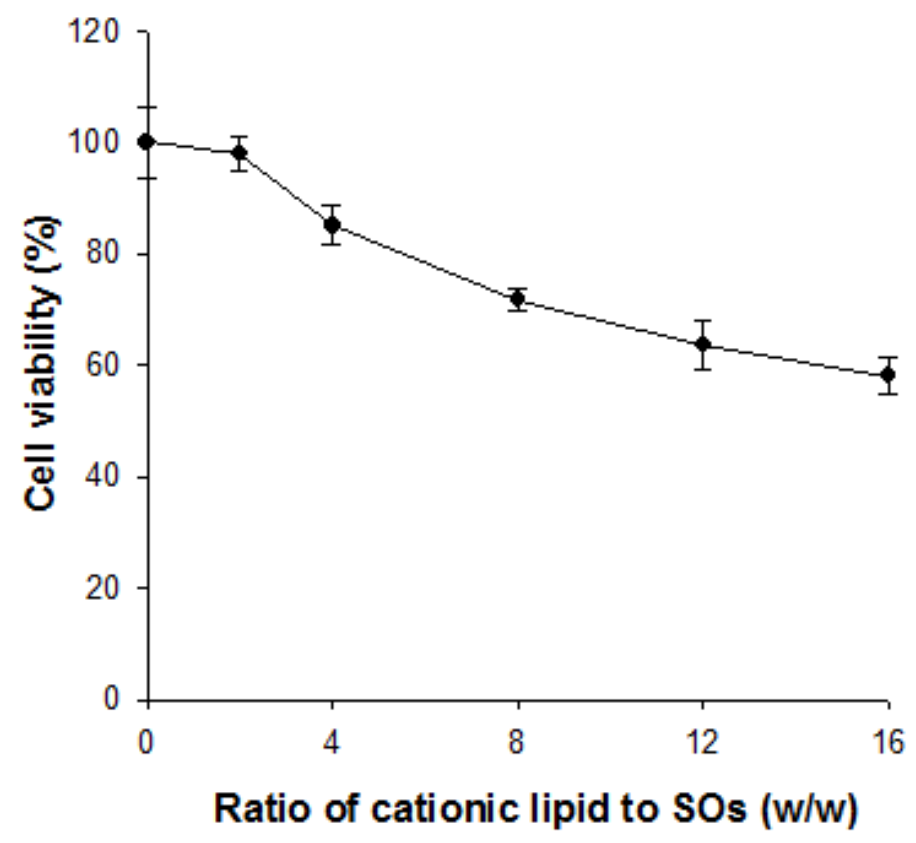

b

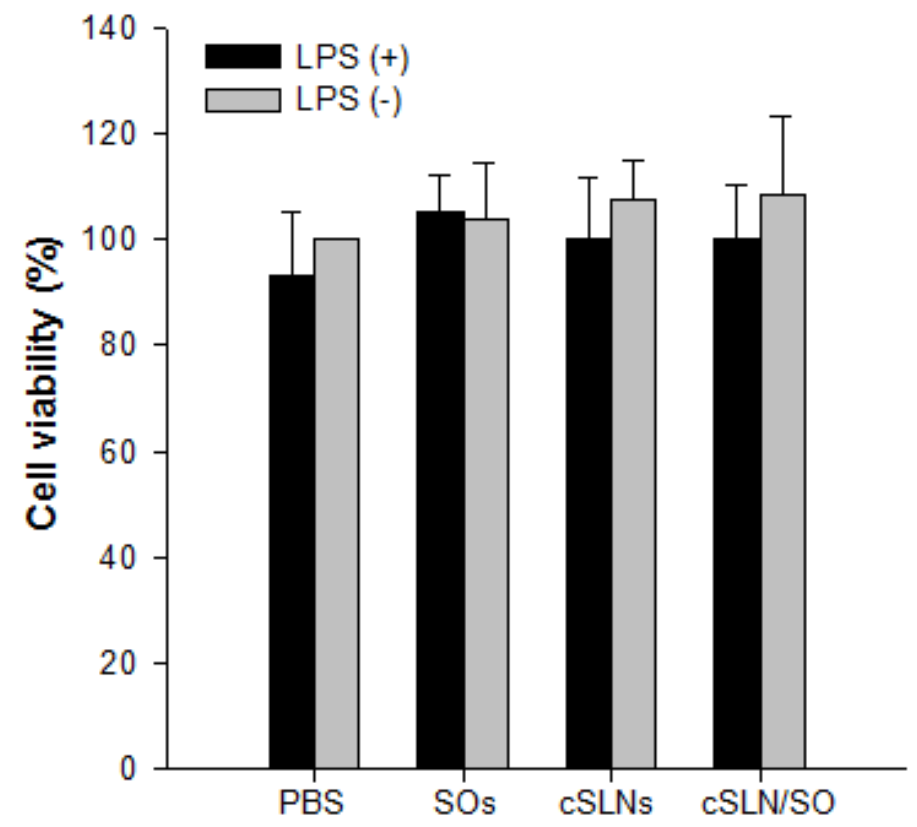

Figure 6 Cell viability of the cSLN/SO complexes in RAW264.7 cells. (a) Cell viability of the cSLN mass ratiodependent SO complexes $(n=4)$ and (b) Cell viability of the cSLN/SO complexes in LPS-treated and untreated RAW264.7 cells ( $\mathrm{n}=6$ ). LPS was used to activate RAW264.7 cells. The results are expressed as the mean \pm SD.

We confirmed that the cSLNs were wellformulated and successfully prepared because TC was presented as nano-crystals from the DSC results (Figure 2). The prepared cSLNs were physically stable at $4^{\circ} \mathrm{C}$ for long-term storage up to 24 months with narrow size distribution (P.I. $<$ 0.2) (Figure 3a). In particular, there were no needs to have a further lyophilization for the improvement of physical stabilization of the cSLNs. 
a

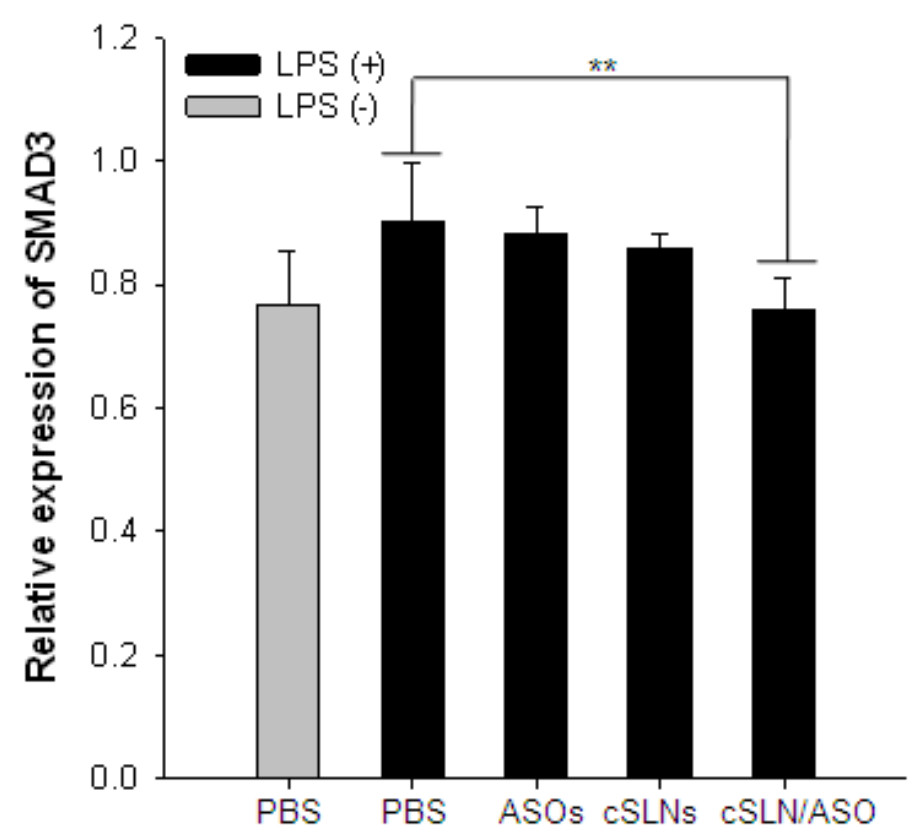

b

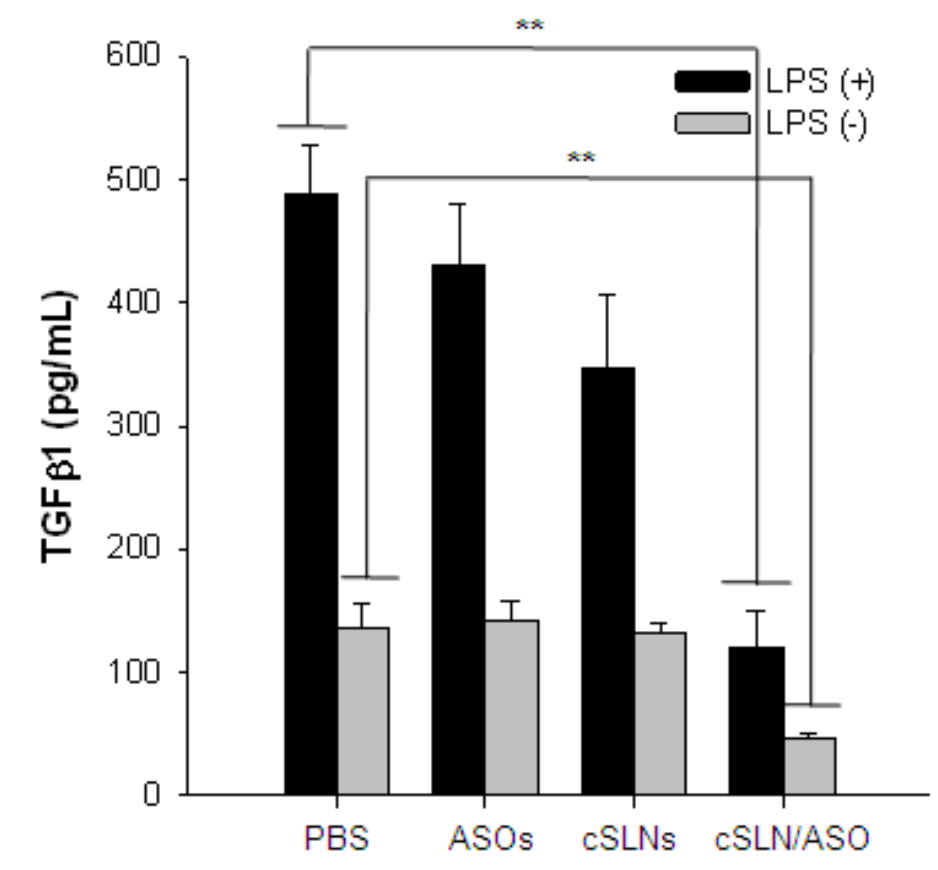

Figure 7 Enhanced intracellular delivery of the SMAD3 ASOs in activated RAW264.7 cells. (a) Modulation of SMAD3 expression in LPS-treated RAW264.7 cells. The band density ratios of SMAD3/ $\beta$-actin are plotted for each group. The results are expressed as the mean $\pm \mathrm{SD}(\mathrm{n}=3)$. (b) Inhibition of TGF $\beta 1$ in LPS-treated and untreated RAW264.7 cells. The results are expressed as the mean $\pm \mathrm{SD}(\mathrm{n}=3)$. ** denotes $p<0.01$. 

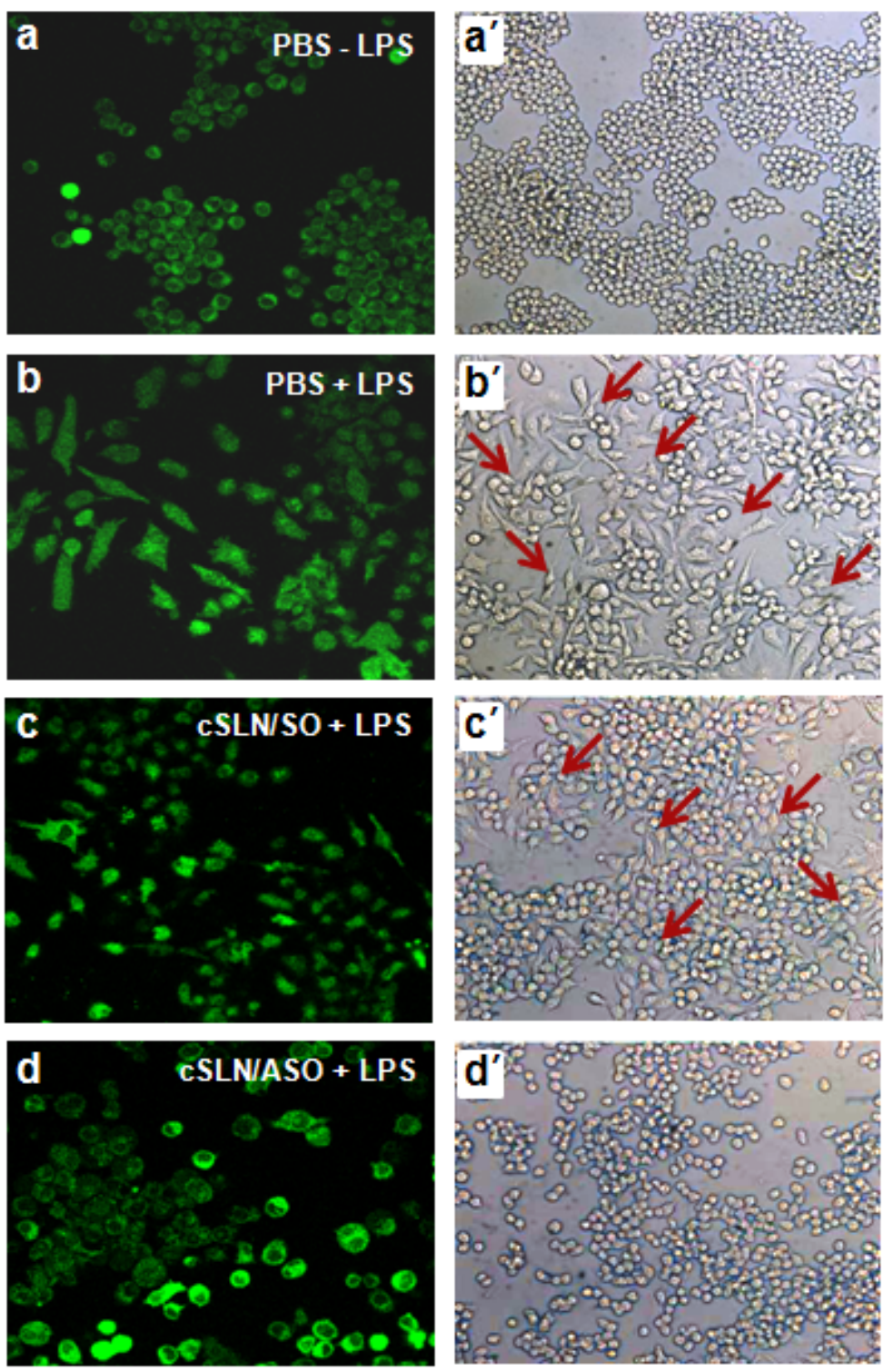

Figure 8 Effect of the cSLN/ASO complexes on the cell shape in LPS-activated RAW264.7 cells. After the treatment of (bb') the PBS (PBS + LPS), (cc') the cSLN/SO complexes (cSLN/SO + LPS), and (dd') the cSLN/ASO complexes (cSLN/ASO + LPS) followed by the LPS stimulation, the morphological change of circle to dendrite was examined in RAW264.7 cells using microscopy. (aa') PBS without LPS (PBS - LPS) was used as a negative control. Greenfluorescence (a-d) and bright-field (a'-d') images were obtained at $200 \times$ and $100 \times$ magnification, respectively. Green fluorescence represents Biodipy ${ }^{\circledR}-\mathrm{C} 5$ stained cells. The red arrows point dendrite-like cells after the LPS stimulation (b'c').

This physical stability of the cSLNs was due to the solid property of the TC core within the particles (22). These cSLNs were nano-sized and positively charged to form a complex with the negatively charged ASOs (Figure 3b). The cSLN/ASO complexes were prepared by the charge-based interaction of the cSLNs and the ASOs, which was confirmed whether the complexes could be formed by the measurement of the size and the zeta potential of the complexes. The decrease of the size of the complexes was explained by the tight binding of the cSLNs and the ASOs to condense. In addition, the increase of the zeta potential of the complexes suggests the interaction of the cSLNs and the ASOs to bind.

For the complex formation, the ASO-bound cSLN complexes over the mass ratio of 4 (cationic lipid to ASOs, w/w) were successfully 
generated with the tight immobilization of the ASOs on the cSLNs confirmed using gel retardation assay (Figure 4a). From the gel retardation of the cSLN/ASO complexes, the charge-based interaction between the cSLNs and the ASOs was confirmed to load the ASOs on the cSLNs. After the successful binding of the ASOs on the surface of the cSLNs, we needed to confirm whether the cSLNs-based ASOs were stable to have a pharmacological activity. Although three sequences of the ASOs at each end were modified by phosphorothioation, those were not completely resistant to enzymatic digestion. The ASOs were readily degraded by nuclease even at the lower concentration of DNase I ( $0.25 \mathrm{U})$ (Figure $4 \mathrm{~b})$. The intracellular activity of the ASOs was highly dependent on the carrier system to protect the ASOs from the enzymatic digestion. In this study, we confirmed that the cSLNs provided the ASOs to resist the enzymatic degradation via the complex formation with the ASOs, which could prevent the interaction between the ASOs and the enzyme to digest (Figure $4 \mathrm{~b}$ ). The protection potential of the cSLNs was comparable to that of Lipofectin ${ }^{\circledR}$.

The cSLNs remarkably enhanced the cellular uptake of the SOs. The cSLN/SO complexes showed the enhanced uptake efficiencies in RAW264.7 cells and NIH-3T3 cells. The uptake efficiencies in RAW264.7 cells were higher than those in NIH-3T3 cells even by 2 -fold (Figure 5a). The differences in the patterns of cellular uptake between cell lines are generally due to the differences in the composition of cell membrane and the mechanism of cellular uptake and processing of the cSLN/SO complexes (23). In the case of RAW264.7 cells, the cSLN/SO complexes at the ratio of 4 were largely going into cells over $80 \%$, which was higher than the uptake efficiency of Lipofectin ${ }^{\circledR}$-based SO complexes (Figure 5b). This result may explained by the mechanism of the cellular uptake of particular system in macrophages. The nanoparticles, can be internalized into Raw 264.7 cells, murine macrophage cells, by the phagocytosis and/or the endocytosis such as clathrin-mediated endocytosis, clathrin-independent endocytosis/cholesteroldependent endocytosis, and macropinocytosis (24, 25). In particular, the hydrophobic and negative nanoparticles easily achieved the uptake by cells via the phagocytosis (24). In addition, the size was one of factors to control the uptake efficiency of the complexes, which was $150-200 \mathrm{~nm}$ to be easily internalized $(24,25)$. Those were similar to the size and the relatively negative property of the cSLN-based ON complexes at the ratio of 4 to have an enhanced uptake potential compared with other ratio-based complexes (Table 1). Furthermore, the long-term stored cSLN-based SO complexes had a comparable uptake potential to RAW264.7 cells compared with the freshly prepared cSLN-based SO complexes (Figure 5b). From the results, the cSLNs were not only physically stable, but also biologically stable to use for the cellular delivery of the ASOs in terms of the enhanced uptake potential after the longterm storage.

Before checking the intracellular activity of the cSLN/ASO complexes, we examined the mass ratio dependent cytotoxicity of the cSLN/SO complexes to optimize the working concentration of the cSLNs and the cSLN/ASO complexes. The $\mathrm{cSLN} / \mathrm{SO}$ complexes at the ratios of 2 to 4 were acceptable to use showing over $80 \%$ of cell viability in this study (Figure 6a). Next, we confirmed the cell viability of the several groups, treated with the SOs, the cSLNs, and the cSLN/SO complexes. From the results, no cytotoxicity was detectable at both conditions of cells with and without LPS (Figure 6b). It suggests that the cSLNs can be an effective carrier to transfer the ASOs into the cells without considerable toxicity. Moreover, the given ratio of 4 in the cSLN-based complexes was consistently exposed as an optimized ratio for further study.

RAW264.7 cells, mouse macrophage cells, release many inflammatory molecules after the LPS stimulation such as interleukins and cytokines (26). These cells have been used to screen anti-inflammatory therapeutics and to study the mechanism of action of antiinflammatory agents as an in vitro cell model (26, 27). In particular, the TGF $\beta / S M A D$ signaling pathway is extensively activated in RAW264.7 cells after the LPS stimulation (28). This pathway was shown to be profoundly related to inflammatory diseases (e.g., fibrosis, cancer, etc.) in monocytes/macrophages extended to an immunological disorder (3). Therefore, RAW264.7 cells were selected to confirm the intracellular activity of the cSLN-based SMAD3 ASOs and to monitor the level of TGF $\beta 1$ after the treatment of the cSLN/SMAD3 ASO complexes.

We found that the cSLN-based SMAD3 ASOs not only significantly inhibited the expression of SMAD3 (Figure 7a), but also suppressed the level of TGF $\beta 1$ indirectly (Figure 7b), which was based on the feedback mechanism of signaling pathway $(1,29)$. The inhibition of SMAD3 as well as the reduction of TGF $\beta 1$ would enhance the antiinflammatory therapeutic effects of the formulation. As the SMAD3 ASOs additionally 
reduced the TGF $\beta 1$, those could maximize the pharmacological activity at the intracellular level of the eventual sites. The profound cellular effects of the SMAD3 ASOs provide potential tools for screening and developing anti-inflammatory therapeutics. In addition, these effects of the SMAD3 ASOs were confirmed to observe the physiology of RAW264.7 cells blocking the morphological changes of the cells with the LPS stimulation (Figure 8). The cSLN/SMAD3 ASO complexes prevented the LPS-stimulated morphological changes of the RAW264.7 cells compared with the LPS-treated PBS and the LPPS-treated cSLN/SO complexes. From the results, our data consistently showed the suppression of SMAD3 in the stimulated RAW264.7 cells after the treatment of the cSLN/ASO complexes suggesting the therapeutic potential of the cSLN-based SMAD3 ASOs.

Although a limitation of this study remains that the several signaling of inflammatory response in RAW264.7 cells was not examined after the treatment of the cSLN/ASO complexes, this study still has several valuable scientific results as follows - (i) the prolonged stability of the cSLNs without further freeze-drying step, (ii) the enhanced uptake efficiency of the ASOs even at the low mass ratio of the cSLNs, and (iii) the specific blocker of signal pathway using the ASOs, which have a therapeutic potential to use for the prevention of inflammation. In conclusion, the cSLN-based SMAD3 ASOs can have a therapeutic potential to alleviate the inflammation process linked to TGF $\beta / \mathrm{SMAD} 3$ pathway. This strategy could be developed for the prevention and the modulation of the inflammation process in the severe immunological disorders. We anticipate that this long-term stable formulation of the cSLNs can be widely used for the in vitro and in vivo delivery of the ASOs based on the enhanced uptake potential, and can be extended to use for the intracellular delivery of plasmid DNA and siRNA.

\section{REFERENCES}

1. Bonniaud P, et al. TGF-beta and Smad 3 signaling link inflammation to chronic fibrogenesis. J Immunol, 2005; 175(8):5390-5395.

2. Wynn TA. Fibrotic disease and the $\mathrm{T}(\mathrm{H}) 1 / \mathrm{T}(\mathrm{H}) 2$ paradigm. Nat Rev Immunol, 2004; 4(8):583-594.

3. Flanders KC. Smad3 as a mediator of the fibrotic response. Int J Exp Pathol, 2004; 85(2):47-64.

4. Zhao J, et al. Smad3 deficiency attenuates bleomycin-induced pulmonary fibrosis in mice. Am J Physiol Lung Cell Mol Physiol, 2002; 282(3):L585-L593.
5. Wynn TA. Common and unique mechanisms regulate fibrosis in various fibroproliferative diseases. J Clin Invest, 2007; 117(3):524-529.

6. Stein CA, Cheng YC. Antisense oligonucleotides as therapeutic agents-is the bullet really magical? Science, 1993; 261(5124):1004-1012.

7. Crooke ST. Progress in antisense technology. Annu Rev Med, 2004; 55:61-95.

8. Temsamani J, Pari GS, Guinot P. Antisense approach for the treatment of cytomegalovirus infection. Expert Opin Investig Drugs, 1997; 6(9):1157-1167.

9. Zuhorn IS, Engberts, JB, Hoekstra D. Gene delivery by cationic lipid vectors: overcoming cellular barriers. Eur Biophys J, 2007; 36(45):349-362.

10. Junghans $\mathrm{M}$, et al. Cationic lipid-protamine-DNA (LPD) complexes for delivery of antisense c-myc oligonucleotides. Eur J Pharm Biopharm, 2005; 60(2):287-294.

11. Semple SC, et al. Rational design of cationic lipids for siRNA delivery. Nat Biotech, 2010; 28(2):172176.

12. Koynova R, Tenchov B. Recent patents in cationic lipid carriers for delivery of nucleic acids. Recent Pat DNA Gene Seq, 2011; 5(1):8-27.

13. Bondi ML, et al. Novel cationic solid-lipid nanoparticles as non-viral vectors for gene delivery. J Drug Target, 2007; 15(4):295-301.

14. del Pozo-Rodriguez A, et al. Solid lipid nanoparticles: formulation factors affecting cell transfection capacity. Int J Pharm, 2007; 339(12):261-268.

15. del Pozo-Rodríguez A, et al. Short- and long-term stability study of lyophilized solid lipid nanoparticles for gene therapy. Eur J Pharm Biopharm, 2009; 71(2):181-189.

16. Choi SH, et al. Novel cationic solid lipid nanoparticles enhanced p53 gene transfer to lung cancer cells. Eur J Pharm Biopharm, 2008; 68(3):545-554.

17. Barteau B, et al. Physicochemical parameters of non-viral vectors that govern transfection efficiency. Curr Gene Ther, 2008; 8(5):313-323.

18. Madeira C, et al. Fluorescence methods for lipoplex characterization. Biochim Biophys Acta, 2011; 1808(11):2694-2705.

19. Simberg D, et al. DOTAP (and other cationic lipids): chemistry, biophysics, and transfection. Crit Rev Ther Drug Carrier Syst, 2004; 21(4):257317.

20. Tamaddona AM, Mozhgan Nikravesha HN. Effect of Helper Lipids on Stability and Transfection Activity of Lyophilized Lipoplex Formulations of Antisense and DOTAP Nanoliposomes. Iranian J Pharm Sci, 2011; 7(2):79-87.

21. Muller RH, Mader K, Gohla S. Solid lipid nanoparticles (SLN) for controlled drug delivery a review of the state of the art. Eur J Pharm Biopharm, 2000; 50(1):161-177.

22. Lim SJ, Kim CK. Formulation parameters determining the physicochemical characteristics of 
solid lipid nanoparticles loaded with all-trans retinoic acid. Int J Pharm, 2002; 243(1-2):135-146.

23. Romoren $\mathrm{K}$, et al. Transfection efficiency and cytotoxicity of cationic liposomes in salmonid cell lines of hepatocyte and macrophage origin. Biochim Biophys Acta, 2004; 1663(1-2):127-134.

24. Sheng $Y$, et al. In vitro macrophage uptake and in vivo biodistribution of PLA-PEG nanoparticles loaded with hemoglobin as blood substitutes: effect of PEG content. J Mater Sci: Mater Med, 2009; 20:1881-1891.

25. Lin PJ, et al. Influence of cationic lipid composition on uptake and intracellular processing of lipid nanoparticle formulations of siRNA. Nanomedicine, 2012; in press.

26. Wang SY, et al. Inhibitory effect of activin A on activation of lipopolysaccharide-stimulated mouse macrophage RAW264.7 cells. Cytokine, 2008; 42(1):85-91.

27. Frazier-Jessen MR, et al. NF-kappaB elements contribute to junB inducibility by lipopolysaccharide in the murine macrophage cell line RAW264.7. FEBS Lett, 2002; 513(2-3):203207.

28. Werner F, et al. Transforming growth factor-beta 1 inhibition of macrophage activation is mediated via Smad3. J Biol Chem, 2000; 275(47):3665336658.

29. Latella $G$, et al. Targeted disruption of $\operatorname{Smad} 3$ confers resistance to the development of dimethylnitrosamine-induced hepatic fibrosis in mice. Liver Int, 2009; 29(7):997-1009. 